\title{
459. Paul Rabe, Über die Umwandlung des Narkotins in Nornarcein; ein Beitrag zur Kenntnis der Chinatoxine ${ }^{1}$ ).
}

\author{
[Aus dem Chemischen Institut der Universitāt Jena.]
}

(Eingegangen am 8. Juli 1907.)

Wenn man unbefangen das gesamte experimentelle Material, das sich bei der Konstitutionserforschung der Chinaalkaloide im Laufe der Jahrzehnte angehäuft hat, kritisch verwertet, so sind nicht nur zwei, wie früher an anderer Stelle ${ }^{2}$ ) ansgeführt wurde, sondern vier Formu-

I.

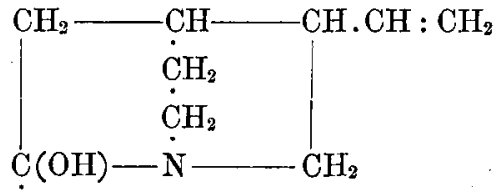

$\mathrm{CH}_{2} . \mathrm{C}_{9} \mathrm{H}_{6} \mathrm{~N}$
II.

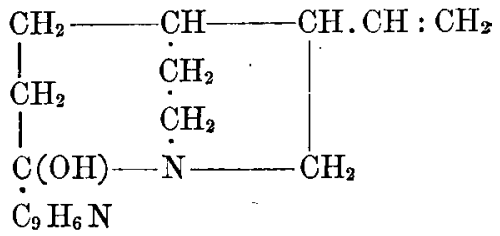

III.

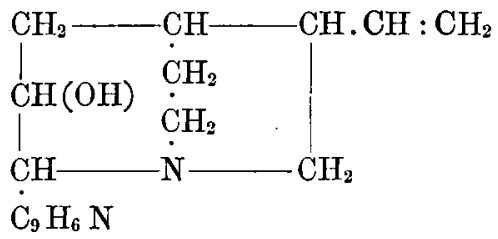

lierungen für das Cinchonin denkbar. Hiervon halte ich die letzte für die richtige: sie erklärt den Übergang von Cinchonin in Cinchen und weiter die Hydrolyse des Cinchens in Lepidin und Merochinen ${ }^{3}$ ), sie entspricht dem Zerfall von Isonitrosocinchotoxin in Cinchoninsäure

1) Für den Ausdruck »Toxine« werde jch in Zukunft nach einem Vorschlage von Hrn. Professor P. Jacobson zur Vermeidung von Verwechselungen den Ausdruck $\gg \mathrm{Ch}$ inatoxine gebrauchen.

$\Rightarrow$ Ann. d. Chem. 350, 180 [1906]. Man vergleiche auch diese Berichte 40, 2013 [1907]. Im Referat dieser Arbeit, Chem. Zentralbl. 1907, II, 74, ist der Inhalt einer Anmerkung infolge eines Versehens entstellt wiedergegeben. Es lautet hier: „Von den beiden Möglichkeiten, das Cinchonin als sekundären, $\mathrm{C}_{9} \mathrm{II}_{6} \mathrm{~N} . \mathrm{CH}(\mathrm{OH}) \cdot \mathrm{C}_{9} \mathrm{H}_{14} \mathrm{~N}$, oder als tertiären Alkohol, $\mathrm{C}_{9} \mathrm{H}_{6} \mathrm{~N}$. C $(\mathrm{OH}): \mathrm{C}_{9} \mathrm{H}_{15} \mathrm{~N}$, aufzufassen, scheint die letztere die richtige zu sein." Statt der letzten Worte muß es heifen: "scheint die erstere die richtige zu sein«.

3) W. Koenigs, diese Berichte 29, 2669 [1890]; 27, 900, 1501 [1894]; Ann. d. Chem. 347, 180 [1906]. 
und das Nitril des Merochinens ${ }^{1}$ ), sie verdeutlicht endlich die weitgehende Analogie, die zwischen der Lmlagerung der Chinabasen in die Chinatoxine einerseits und der Verwandlung von Narkotin in Narcein, ron Hydrastin in Methylhydrastein und ron Oxykodein in Ketodihydromethylmorphimethin andererseits besteht ${ }^{2}$ ).

Es erscheint indessen keineswegs ausgeschlossen, daß bei den ersten Eingriffen in das Molekül einer Chinabase noch völlig unbekannte Umlagerungen eintreten könnten. Ich habe daher zur weiteren Begründung der Formel IV das Studium der Oxydationsvorgänge wieder aufgenommen ${ }^{3}$ ). Außerdem halte ich eine vergleichende Untersuchung über den Mechanismus der oben erwähnten Umwandlungen von Hydraminen in Ketonbasen für notwendig ${ }^{4}$ ).

In der vorliegenden Mitteilung soll über die Umwandlung des Narkotins in eine Ketonbase berichtet werden.

1) Rabe, Ann. d. Chem. 350, 180 [1906]; Rabe und Ritter, diese Berichte 38, 2770 [1905] Dic Auffindung und Erklärung dieses Zerfalles bedeutet ebenso wie die Entdeckung der Chinatoxine durch v. Miller und Rhode einen Abschnitt in der neueren Bearbeitung der Chinalkaloide. Die \&lurch jenen Zerfall notwendig gewordene Abänderung der früher von v. Miller und Rhode vertretenen Ansicht über die Stellung der Carbonylgruppe in den Chinatoxinen hat zu neuen C̈berlegungen und Versuchen geführt. Hieran haben sich auch K. Bernhart und J. Jbele, diese Berichte 40, 648, 2873 [1907], einerseits, G. Rhode und A. Antonaz, diese Berichte 40, 2329 [1907], andererseits beteiligt. Sie haben sich bemüht, die von mir bewiesene Cinchotoxinformel durch weitere Versuche zu stützen. Auf diese Arbeitẹ werde ich später in einer zusammenfassenden Abhandlung zurückkommen.

2) Diese Berichte 40, 2013 [1907], Fußnote 2.

$\left.{ }^{3}\right)$ Es wurde auch schon eịe neue Verbindung, ein Einwirkungsprodukt ron Salpetersäure auf Cinchonin beschrieben (Rabe und Ackermann, diese Berichte 40, 2016 [1907]). Ein anderes Oxydationsprodukt haben wir mit Hilie von Chromsäure gewonnen. Es krystallisiert aus verdünntem Alkohol in rhombischen Blättchen oder Nädelchen von schwach gelblicher Farbe. Schmp. 126-1270. Die Verbindung besitzt die Zusammensetzung $\mathrm{C}_{19} \mathrm{H}_{20} \mathrm{ON}_{2}$; sie unterscheidt sich rom Cinchonin in charalteristischer Weise durch ihre Fähigkeit, ein Oxim zu bilden. Hierüber wird Hr. Ackermann in seiner Dissertationsschrift Näheres mitteilen. Versuche zur Gewinnung der entsprechenden Verbindungen aus den andern Chinaalkaloiden und ans dem Cinchonin sind von den HHrn. Fr. Braasch und W. Naumann in Angriff genommen.

4) IIierzu gelı̈ren auch Versuche zur Rückverwandlung der Chinatoxine in bicyclische Verbindungen. Solche Versuche habe ich schon vor Jalıren (diese Berichte 37, 1674 [1904]) im Anschluß an die Arbeiten über die Unlagerung von 1.5-Diketonen in cyclische Ketonalkohole ausgefülurt, allerdings mit negativem Erfolg. Siehe auch W. Koenigs, diese Berichte 40, 2875 [1907]. 
Das Narkotinjodmethylat geht nach den Erfahrungen Rosers 1 ) durch Erhitzen mit Alkalien in ein ancleres Opiumalkaloid, das Narcein, über. Diese Reaktion ist ein Analogon zur C̈berführung des Cinchoninjodmethylats in Methylcinchotoxin: in beiden Fällen wird die Ringöffuung begleitet ron dem Verschwinclen eines alkoholischen $\mathrm{Hy}$ droxyls und dem gleichzeitigen Auftreten einer Ketongruppe; beim Narkotin geht dieser Ketonbildung natürlicherweise eine hydrolytische Aufspaltung des Lactonringes voraus.

Eine zweite vollständig analoge Veränderung erfährt das Cinchonin beim Kochen mit verdünnter Essigsäure $\left.{ }^{3}\right)$ :

\section{Cinchonin $\longrightarrow$ Cinchotoxin.}

Es fragt sich, ob unter diesen Bedingungen auch aus Narkotim eine Ketonbase hervorgeht. Das ist in der Tat der Fall. Aber neben der Ketonbase treten noch andere Produkte auf, so daß hier verwickeltere Verhältnisse wie bei den Chinaalkaloiden vorliegen. Das Resultat der bisher angestellten Versuche läßt sich kurz durch folgendes Schema

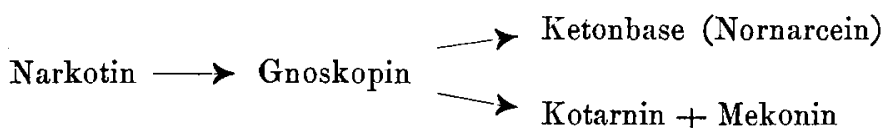

veranschaulichen.

Als erstes Reaktionsprodukt erscheint das Gnoskopin, das von Smith ${ }^{3}$ ) bei der Aufarbeitung der Opiummutterlaugen aufgefunden und später als ein Isomeres des Narkotins erkannt worden ist. Über sein optisches Verhalten habe ich in der Literatur keine Angaben finden können. Es ist inaktiv und dürfte demnach durch Racemisierung aus dem Mutteralkaloid entstehen. Um diese Auffassung zu stützen, soll das Gnoskopin nach verschiedenen Richtungen hin genaner untersucht werden. Erst nach Abschluß dieser Versuche wird man der Frage näher treten können, ob das Gnoskopin wirklich, wie angenommen, die Rolle eines $Z$ wischenprodukts spielt. Daran ist aber kaum zu zweifeln, da reines Gnoskopin beim Behandeln mit verdünnter Essigsäure sowohl Ketonbase wie Kotarnin und Mekonin liefert,

Neben dem Gnoskopin entsteht unter Aufnahme von einem Molekül Wasser eine Ketonbase. Sie kann leicht in das Jodmethylat des

1) Ann. d. Chem. 247, 167 [1888]. Freund und Frankforter, Anr. d. Chem. 277, 57 [1893].

2) v. Miller und lhode, diese Berichte 27, 1187, 1279 [1894]; 28, 1056 [1895].

3) Pharm. Journ. Trans. [3] 9, 82 [1878]. 
Narceinmethylesters übergeführt werden und ist dementsprechend wohl zweckmäßig als Nornarcein

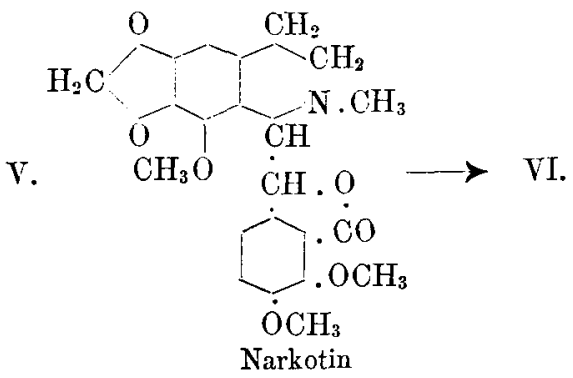

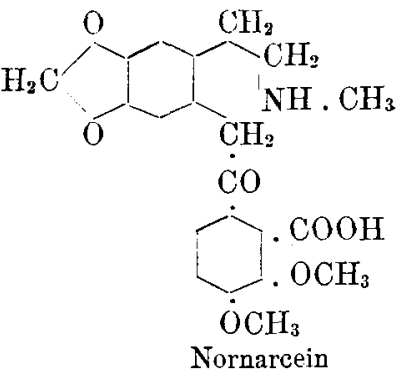

$\mathrm{zu}$ bezeichnen.

Endlich treten unter den Reaktionsprodukten, wie mau von vornherein erwarten mußte, die bekannten Bruchstiicke des Narkotins, Kotarnin und Mekonin, auf. Bei ihrer Bildung ist das Nornarcein nicht direkt beteiligt. Denn, wie durch einen besonderen Versuch festgestellt wurde, wird das Nornarcein durch verdünnte Essigsäure im Unterschiede zu Narkotin und Gnoskopin nicht weiter gespalten; es gleicht daher in dieser Beständigkeit dem Cinchotoxin.

Der Vergleich der Spaltungen beim Narkotin und Cinchonin hat also ergeben: Beide Alkaloide erleiden unter dem Einflusse von verdünnter Essigsäure die Umwandlung in eine Ketonbase. Daneben vollzieht sich allein beim Narkotin noch eine zweite tiefer eingreifende Reaktion, der Zerfall in zwei Bruchstücke. Keine Beobachtung spricht gegen die Annahme, daß die im Narkotin oder richtiger in seinen Alkalisalzen vorhandene Atompruppe

$$
>\mathrm{N} \text {. CH. } \mathrm{CH}(\mathrm{OH}) \text {. }
$$

auch im Cinchonin vorkommt.

$$
\text { Experimentelles. }
$$

Einwirkung von verdünter Essigsäure auf Narkotin.

$43 \mathrm{~g}$ Narkotin wurden mit $95 \mathrm{~g}$ Eisessig und $500 \mathrm{ccm}$ Wasser 72 Stunden bei einer Ölbadtemperatur von $105-110^{\circ}$ erhitzt. Die erkaltete Reaktionsflüssigkeit wurde mit Natronlauge $(1: 3)$ bis zur neutralen Reaktion versetzt. Es schied sich neben unverändertem Narkotin ein Gemenge von Gnoskopin, von dem bisher noch nicht beschriebenen Nornarcein und von Mekonin ab. Die Trennung dieser vier Verbindungen ist keine ganz leichte; sie läßt sich auf Grund folgender Eigenschaften der einzelnen Substanzen bewerkstelligen. Nur Nornarcein und Mekonin lösen sich bei Zimmertemperatur in Normalnatronlauge. Aus dieser Lösung fällt Kohlensäure allein das 
Nornarcein, während sich das Mekonin als Lacton auf Zusatz von Mineralsäure zur Mutterlauge allmäblich abscheidet. Die in verdünntem Alkali unlöslichen Produkte Narkotin und Gnoskopin unterscheiden sich ron einander durch ihre Löslichkeit in Alkohol, da das Gnoskopin selbst von kochendem Alkohol nur wenig aufgenommen wird und das Narkotin sich in leißem Alkohol relativ leicht, in kaltem schwer löst.

Die oben erwälnte neutrale, von den beschriebenen vier Verbindungen befreite Reaktionsflüssigkeit trübt sich durch Zusatz von überschüssiger Natronlauge $(1: 1)$ milchig und allmählich setzt sich das Kotarnin in Form derber Krystalle ab.

Es wurden so erhalten aus $43 \mathrm{~g}$ Narkotin:

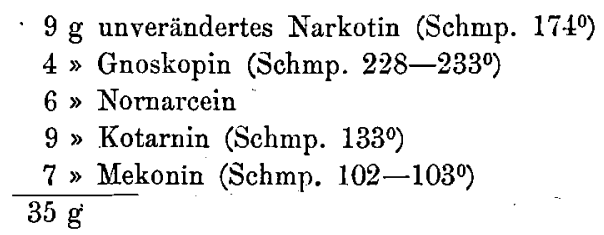

Der Verlust von ca. $8 \mathrm{~g}$ erklärt sich hauptsächlich durch Bildung harziger Substanzen.

$$
\text { Nornarcein, } \mathrm{C}_{22} \mathrm{H}_{25} \mathrm{O}_{8} \mathrm{~N} \text {. }
$$

Diese bisher nicht beschriebene Ketonbase (Formel VI) ist optisch inactiv. Sie gleicht vollkommen dem Narcein. Aus ihrer alkalischen Lösung wird sie durch Kohlensäure in farblosen, weichen, seidenglänzenden, verfilzten Nädelchen abgeschieden, die lufttrocken $3 \mathrm{Mol}$. Krystallwasser enthalten und in diesem Zustande auffallenderweise keinen konstanten Schmelzpunkt zeigen: verschiedene Präparate schmolzen neben einander am gleichen Thermometer erhitzt zwischen $205^{\circ}$ und $222^{\circ}$ unter Zersetzung. Beim Erhitzen auf $105^{\circ}$ gibt die krystallwasserhaltige Verbindung $3 \mathrm{Mol}$. Wasser ab und dabei erniedrigt sich der Zersetzungspunkt auf $147^{\circ}$. Die Substanz ist dann äußerst hygroskopisch. Beide Substanzen lösen sich spielend in siedendem Alkohol, aber schon nach wenigen Sekunden scheidet sich eine höher schmelzende, wasser- und alkoholfreie Modifikation in Form prismatischer Krystalle ab, die scharf bei $229^{\circ}$ ebenfalls unter Zersetzung schmelzen. Worauf diese merkwürdige Änderung der Eigenschaften beim Umlösen aus Alkohol beruht, läßt sich noch nicht sicher angeben. Auch das aus Alkohol gewonnene Präparat besitzt die Formel $\mathrm{C}_{22} \mathrm{H}_{25} \mathrm{O}_{8} \mathrm{~N}$, löst sich in Alkalien und liefert beim Wiederausfällen mittels Kohlensäure die ursprüngliche krystallwasserhaltige Modifikation zurück. Diese Rückverwandlung kann auch durch einfaches Umkrystallisieren aus Wasser erreicht werden. 
$0.8118 \mathrm{~g}$ lufttrockne Sbst. verloren bei mehrstündigem Erhitzen auf $105^{\circ}$ $0.0917 \mathrm{~g} \mathrm{H}_{2} \mathrm{O}$.

$\mathrm{C}_{22} \mathrm{H}_{25} \mathrm{O}_{8} \mathrm{~N}+3 \mathrm{H}_{2} \mathrm{O}$. Ber. $\mathrm{H}_{2} \mathrm{O}$ 11.12. Gef. $\mathrm{H}_{2} \mathrm{O}$ 11.30.

$0.2280 \mathrm{~g}$ der bei $105^{\circ}$ getrockneten Sibst.: $0.5102 \mathrm{~g} \mathrm{CO}_{2}, 0.1208 \mathrm{~g} \mathrm{H}_{2} \mathrm{O}$. - $0.2035 \mathrm{~g}$ Sbst.: $7.5 \mathrm{ecm} \mathrm{N}\left(24^{\circ}, 748 \mathrm{~mm}\right) .-0.2035 \mathrm{~g}$ ans Alkohol krystallisierte Sbst.: $0.4579 \mathrm{~g} \mathrm{CO}_{2}, 0.1063 \mathrm{~g} \mathrm{H}_{2} \mathrm{O}$.

$$
\begin{aligned}
& \mathrm{C}_{22} \mathrm{H}_{25} \mathrm{O}_{8} \mathrm{~N} \text {. Ber. C 61.25, } \quad \mathrm{H} 5.80, \quad \mathrm{~N} 3.25 \text {. } \\
& \text { Gef. 》 } 61.03,61.37, \text { 》 } 5.91,5.84, 》 3.43 \text {. }
\end{aligned}
$$

Das Nornarceinchlorhydrat scheidet sich aus 20-proz. Salzsäure in farblosen, prismatischen Stäbchen vom Schmp. $144^{\circ}$ aus. Die über Schwefelsäure getrocknete Substanz enthält 1 Mol. Krystallwasser. Sie verliert dasselbe bei $105^{\circ}$ and ist dann sehr hygroskopisch.

$0.8355 \mathrm{~g}$ Sbst. verloren bei $105^{\circ}$ nach einstündigem Erhitzen $0.0323 \mathrm{~g} \mathrm{H}_{2} \mathrm{O}$. $\mathrm{C}_{29} \mathrm{H}_{26} \mathrm{O}_{8} \mathrm{~N} \mathrm{Cl}+\mathrm{H}_{2} \mathrm{O}$. Ber. $\mathrm{H}_{2} \mathrm{O}$ 3.71. Gef. $\mathrm{H}_{2} \mathrm{O} 3.87$.

$0.2544 \mathrm{~g}$ bei $105^{\circ}$ getrocknete Sbst.: $0.5248 \mathrm{~g} \mathrm{CO}_{2}, 0.1306 \mathrm{~g} \mathrm{H}_{2} \mathrm{O}$. 0.3642 g Sbst.: $0.1243 \mathrm{~g} \mathrm{AgCl}$.

$$
\begin{aligned}
& \mathrm{C}_{22} \mathrm{H}_{26} \mathrm{O}_{8} \mathrm{NCl} \text {. Ber. C 56.53, H 5.57, } \mathrm{Cl} 7.50 \text {. } \\
& \text { Gef. »56.26, 》5.70, 》7.35. }
\end{aligned}
$$

Das Oxim des Nornarceins erhält man bei der Einwirkung von Hydroxylaminchlorhydrat auf beide Modifikationen der Ketonbase zunächst in Form des salzsauren Oximanhydrids. Dieses schmilat, aus Alkohol umkrystallisiert, bei $138^{\circ}$, enthält $1 \mathrm{Mol}$. Krystallalkohol iußerst fest gebunden und färbt sich im direkten Lichte gelb.

$0.9364 \mathrm{~g}$ lnfttrockne Sibst. verloren nach 10-stïndigem Errhitzen auf $10 \tilde{5}^{0}$ $0.0894 \mathrm{~g}$ Alkohol.

$\mathrm{C}_{22} \mathrm{H}_{25} \mathrm{O}_{7} \mathrm{~N}_{2} \mathrm{Cl}+\mathrm{C}_{2} \mathrm{H}_{5} \mathrm{OH}$. Ber. $\mathrm{C}_{2} \mathrm{H}_{5} \mathrm{OH}$ 9.10. Gef. $\mathrm{C}_{2} \mathrm{H}_{5} \mathrm{OH} 9.54$

$0.2246 \mathrm{~g}$ bei $105^{\circ}$ getrocknete Sbst.: $0.4668 \mathrm{~g} \mathrm{CO}_{2}, 0.1048 \mathrm{~g} \mathrm{H}_{2} \mathrm{O}$. -

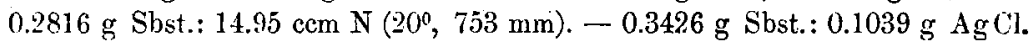

$$
\begin{aligned}
& \mathrm{C}_{22} \mathrm{H}_{25} \mathrm{O}_{7} \mathrm{~N}_{2} \mathrm{Cl} \text {. Ber. C 56.91, H 5.38, N 6.04, Cl 7.64. } \\
& \text { Gef. 》56.68, 》5.40, 》6.13, 》7.50. }
\end{aligned}
$$

Ans der wäßrigen Lüsung seines salzsauren Salzes läßt sich das Oximanhydrid durch die berechnete Menge Natronlauge als gelbliche, klebrige Masse abscheiden, die jedoch schon nach kurzer Zeit unter Aufnahme von Wasser als Oxin in Lösung geht. Ans dieser Lösung konnte das Oxim wegen seiner Schwerlöslichkeit in Alkohol und Leichtlösliclikeit in Wasser nicht frei von Kochsalz gewonnen werden. Die Reindarstellung gelingt abes. bei ler Umsetzung des salzsauren Oximanhydrids mit der berechneten Menge Silbercarbonat. Das Oxim wird aus 80-proz. Alkohol in Form rhombischer Blättchen erhalten und schmilzt bei $171^{\circ}$. Beim Erwärmen auf $108^{\circ}$ geht dir Substanz im Unterschied zu den Beobachtungen Freund's beim Nareeinoxim") nicht in das Anhydrid äber.

3) Freund und Frankforter, Ann. d. Chem. 277, 53 [1893]. 
$0.1583 \mathrm{~g}$ bei $108^{\circ}$ getrocknete Sbst.: $0.3395 \mathrm{~g} \mathrm{CO}, 0.0832 \mathrm{~g} \mathrm{H}_{3} \mathrm{O}$.

$\mathrm{C}_{22} \mathrm{H}_{36} \mathrm{O}_{7} \mathrm{~N}_{2}$. Ber. C 59.19, H 5.83 .

Gef. »58.79, 》5.91.

Überführung des Nornarceins in das Jodmethylat des Narceinmethylesters ${ }^{1}$ ).

1 Mol. Nornarcein rom Schmp. 1470, 2 Mol. Natriummethylat und überschüssiges Jodmethyl wurden in methylalkoholischer Lösung 3 Stunden erhitzt. Nach dem Eindampfen wurde der Rückstand in heißem Wasser aufgenommen. Beim Erkalten schied sich das Jodmethylat zunächst in Form eines Öles aus, das bei längerem Stehen in krystallinischen Zustand übergeht. Nach dem Umkrystallisieren aus Alkohol schmolz das Jodmethylat bei $207-208^{\circ}$ ebenso wie ein aus Narcein hergestelltes Vergleichspräparat. Der Schmelzpunkt der Mischprobe lag ebenfalls scharf bei $208^{\circ}$.

$0.2898 \mathrm{~g}$ bei $105^{\circ}$ getrocknete Sbst.: $0.5262 \mathrm{~g} \mathrm{CO}_{2}, 0.1494 \mathrm{~g} \mathrm{H}_{2} \mathrm{O}$. $0.4340 \mathrm{~g}$ Sbst.: $0.1633 \mathrm{~g} \mathrm{AgJ}$.

$$
\begin{aligned}
\mathrm{C}_{25} \mathrm{H}_{32} \mathrm{O}_{8} \mathrm{NJ} . & \text { Ber. C 49.91, H 5.32, J } 21.13 . \\
& \text { Gef. » 49.52, » 5.57, " } 21.12 .
\end{aligned}
$$

Verhalten des Nornarceins gegen verdünnte Essigsäure.

Um die Beständigkeit der Ketonbase gegen verdünnte Essigsäure zu prüfen, wurden $3 \mathrm{~g}$ Base unter gleichen Reaktionsbedingungen wie oben das Narkotin 72 Stunden erhitzt. Aus der Reaktionsflüssigkeit wurden $2.7 \mathrm{~g}$ unverändert wiedergewonnen. Trotz sorgiältiger Durcharbeitung konnten außer harzigen Produkten weder Mekonin noch Kotarnin aufgefunden werden.

Verhalten des Gnoskopins gegen verdiinnte Essigsäure.

3 g Gnoskopin (Schmp. 232-23302)) lieferten unter den beim Narkotin angegebenen Versuchsbedingungen mit verdünnter Essigsäure erhitzt:

1) Freund, diese Berichte 40, 200 [1907]

2) In der Literatur ist für das Gnoskopin der Sehmp. 228" angegeben. Den bisherigen Angaben über diese Base sei hinzugefügt, daß sie optisch inaktiv ist und zweckmäßig in der Weise umkrystallisiert wird, dab man ihre Auflösung in der zureichenden Menge Chloroform mit den mohrfichen Vislumen Alkohol versetzt. Auf diese Weise erhält man ain Produkt, dab bei $232-233^{\circ}$ schmilzt. 
$0.25 \mathrm{~g}$ unverändertes Giloskopin

$0.6 \gg$ Nornarcein

0.8 » Mekonin

0.8 » Kotarnin

Rest: harzige Produkte.

Schlielslich spreche ich Hrn. Ir. W. Schueider für die ausgezeichnete Hilfe bei Ausführuug dieser Arbeit meinen besten L'ank aus.

\section{H. v. Wartenberg: Der Schmelzpunkt des reinen Wolframs.}

(Eingegangen am 9. Juli 1907.)

[Aus dem Physikal.-chem. Institut der Vuiversitait]

(Eingegangen am 8. Juli 1907; vorgetragen in der Sitzung vom Verfasscr.)

Aus der Wolframlampentechnik ist bekannt, daß der Schmelzpunkt des Wolframs sehr hoch liegt, in der Gegend von $3000^{\circ}$. Es erschien wegen dieser abnormen Höhe von Interesse, ihn genauer festzulegen, wozu aber erst eine Schmelzmethode ausgearbeitet werden mußte. Das einzig ${ }^{1}$ ) für solche 'T'emperaturen bekannte Verfahren, das Moissansche, vermeidet nur unter besonderen Kautelen ${ }^{2}$ ) die Carbidbildung und ist zur Beobachtung des Niederschmelzens ungeeignet.

Eine gut arbeitende Methode gewinnt man nun durch Benutzung eines Geiblerschen Rohres mit Wehneltscher Katbode ${ }^{3}$ ). Eine solche aus einem glühenden, mit Erdalkalioxyd bestrichenen Platinblech bestehende Kathode erniedrigt den in gutem Vakuum mehrere Tausend Volt betragenden Kathodenfall auf $1-2$ Volt, so daß man mit Hilie eines Gleichstroms ron z. B. 110 Volt bequem starke Ströme durch das Vakuum senden kann. Der Spannungsalffall konzentriert sich dann fast ganz auf den von der Stromstärke unabhängigen Anodenfall ron 30-40 Volt und den Abfall in der freien Gasstrecke (1-2 Volt $/ \mathrm{cm})$. Da der Anodenfall auf der Oberfläche der Anode aufsitzt, kann man sozusagen eine leliebige Wattzahl auf die beliebig

1) v. Bolton (Ztschr. für Elektrochem. 11, 45 [1905] u. 13, 146 [1907]) gibt ein zum Schmelzen von z. B. Tantal geeignetes Verfahren an unter Benutzung eines Lichtbogens im Vakuum. Die Methode ist jedoch Fabrikgrheimnis und kommt daher hier nicht in Betracht.

2) Mo issan, Compt. rend. 123, 13 [1896].

3) Wehnelt, Ann. Phș. 14, 425 [1904] und 19, 138 [1906]. 